INPLASY

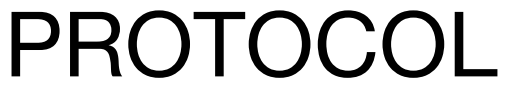

To cite: Liu et al. A metaanalysis of laser peripheral iridoplasty in the treatment of acute angle-closure glaucoma. Inplasy protocol 202180051. doi:

10.37766/inplasy2021.8.0051

Received: 12 August 2021

Published: 12 August 2021

Corresponding author:

Liu Guoying

liuguoying_dd@sina.com

Author Affiliation:

The Sixth Affiliated

Hospital,South China

University of Technology.

Support: None.

Review Stage at time of this submission: Data extraction.

Conflicts of interest:

None declared.

\section{A meta-analysis of laser peripheral iridoplasty in the treatment of acute angle-closure glaucoma}

Review question / Objective: P:Acute angle-closure glaucoma; I:iridoplasty; C:medical treatement; O:Drop in intraocular pressure, IOP control rate; S:randomized controlled trial.

Condition being studied: The acute attack of acute angleclosure glaucoma (PACG) is one of the common emergencies in ophthalmology. It is caused by the sudden increase in intraocular pressure caused by the acute closure of the angle of the chamber. If timely treatment and intervention are not available, it may Will cause irreversible damage to visual function. Drug treatment was a common treatment, but it may bring systemic side effects, and it fails to achieve the purpose of rapid alleviation of the disease. Laser treatment (iridoplasty) achieves the purpose of reducing the intraocular pressure by reopening the angle of the chamber by shrinking the peripheral iris.

INPLASY registration number: This protocol was registered with the International Platform of Registered Systematic Review and Meta-Analysis Protocols (INPLASY) on 12 August 2021 and was last updated on 17 August 2021 (registration number INPLASY202180051).

\section{INTRODUCTION}

Review question / Objective: P:Acute angle-closure glaucoma; I:iridoplasty; C:medical treatement; O:Drop in intraocular pressure, IOP control rate; S:randomized controlled trial.
Condition being studied: The acute attack of acute angle-closure glaucoma (PACG) is one of the common emergencies in ophthalmology. It is caused by the sudden increase in intraocular pressure caused by the acute closure of the angle of the chamber. If timely treatment and intervention are not available, it may Will cause irreversible damage to visual 
function. Drug treatment was a common treatment, but it may bring systemic side effects, and it fails to achieve the purpose of rapid alleviation of the disease. Laser treatment (iridoplasty) achieves the purpose of reducing the intraocular pressure by reopening the angle of the chamber by shrinking the peripheral iris.

\section{METHODS}

Search strategy: (Glaucoma, AngleClosure (MeSH) OR primary angle closure glaucoma OR Angle-Closure Glaucoma OR Angle-Closure Glaucomas OR Glaucomas, Angle-Closure OR Glaucoma OR Uncompensated OR GIaucomas, Uncompensated OR Uncompensated Glaucoma OR Uncompensated Glaucomas OR Glaucoma, Closed-Angle OR ClosedAngle Glaucoma OR Closed-Angle Glaucomas OR Glaucoma, Closed Angle OR Glaucomas, Closed-Angle OR Glaucoma, Uncompensative OR Glaucomas, Uncompensative OR Uncompensative Glaucoma OR Uncompensative Glaucomas OR Glaucoma, Angle Closure OR Angle Closure Glaucoma OR Angle Closure Glaucomas Glaucomas, Angle Closure OR Glaucoma, Narrow-Angle OR Glaucoma, Narrow Angle OR Glaucomas, NarrowAngle OR Narrow-Angle Glaucoma OR Narrow-Angle Glaucomas OR acute angle closure glaucoma OR acute angle-closure glaucoma OR acute closed-angle glaucoma) AND ( iridoplasty OR gonioplasty) AND (randomized controlled trial[Publication Type] OR randomized[Title/ Abstract] OR placebo[Title/Abstract]).

Participant or population: Acute angleclosure glaucoma.

Intervention: Iridoplasty.

Comparator: Medical treatement.

Study designs to be included: Randomized controlled trial.

Eligibility criteria: Inclusion criteria: The research object was the acute attack of acutre ts were patients with acute angleclosure glaucoma in remission, intermittent, pre-clinical, chronic angleclosure glaucoma oangle-closure glaucoma, and the race, age, gender, and time of onset were not limited; Research method: the observation goup was laser peripheral iridoplasty, and the control group was conventional medical treatment; study type : Randomized controlled trial; Outcome indicators(including one of the following): IOP drop after treatment, IOP control rate, angle of opening, degree of corneal edema, pupil diameter. Exclusion criteria: Research subjecr suspected angle closure; The intervention measures in the research method did not match; The research type was non-randomized control Experiments; Reviews, conference reports, animal experiments and repeated literature; Unable to obtain the full text or incomplete data; Low-quality journal studies.

Information sources: PubMed, Ebase, Web of science, Cochrane Library, ClinicalTrials, CBM, CNKI, VIP, Wanfang.

Main outcome(s): Treatment effect:IOP drop after treatment, IOP control rate.

Additional outcome(s): Angle of opening, degree of corneal edema, pupil diameter.

Quality assessment / Risk of bias analysis: Cochrane tool.

Strategy of data synthesis: Heterogeneity was assessed by calculating the 12 statistic and by performing a chi-squared test (assessing the p-value). An $\mathrm{I} 2>50 \%$ was considered to be indicative of significant heterogeneity. Random-effects or fixedeffects models were applied according to the between study heterogeneity.

Subgroup analysis: Subgroup analysis according to the time point or range of the outcome index.

Sensitivity analysis: After deleting the data of any one of the documents, is the result of the merged data of the remaining 
documents greatly deviated from the original.

Country(ies) involved: China.

Keywords: acute angle-closure glaucoma, iridoplasty, effect.

Contributions of each author:

Author 1 - Liu Guoying.

Author 2 - Shao Dongping.

Author 3 - Zhao Liuning. 